\title{
Modelos Evolutivos Complejos en Ciencias Sociales
}

\author{
Antonio Garcia-Olivares R. \\ CSIC - LITEC \\ Zaragoza
}

\section{INTRODUCCIÓN}

La emergencia de la forma y el comportamiento complejos en sistemas compuestos de muchos elementos simples interactuantes es uno de los más fascinantes fenómenos de nuestro mundo y uno de los últimos en ser abordados mediante técnicas matemáticas por la ciencia.

Como hemos visto en otro lugar (García Olivares 1999-a), los programas de investigación relacionados con los sistemas autoorganizativos (Nicolis y Progogine, 1977), la sinergética (Haken, 1987) y los estudios sobre sistemas complejos y vida artificial del Instituto de Santa Fe (Santa Fe, 1999) han proporcionado un conjunto de conceptos que tratan de describir de manera no simplista esa clase de fenómenos que caracterizan a los sistemas complejos. Tales conceptos podrían ser de enorme utilidad como punto de partido heurístico en la descripción y simulación de procesos sociales. Algunos de estos conceptos son los siguientes:

(i) la autoorganización por aparición de atractores en sistemas disipativos abiertos,

(ii) la inestabilidad del sistema autoorganizativo ante cambios en los parámetros ambientales,

(iii) la inestabilidad estructural del sistema autoorganizativo ante la aparición de nuevas interacciones,

(iv) el ensamblaje quimérico de sistemas autoorganizativos en la forma de sistemas que se automantienen,

(v) la coevolución adaptativa de las reglas de interacción utilizadas por sistemas agentes en presencia de otros sistemas agentes. 
En este trabajo vamos a dar forma matemática a algunos de estos conceptos con el fin de ilustrar el modo como pueden generarse los modelos de enorme utilidad heurística para la descripción y simulación de la relación entre procesos microsociales y macrosociales y su co-evolución mutua.

\section{MODERNIZACIÓN DE LA INFLUENCIA MUTUA ENTRE LOS NIVELES MICRO Y MACROSOCIAL}

Vimos en otro lugar (García-Olivares, 1993) (García-Olivares, 1999-b) cómo un buen punto de partida para plantear matemáticamente una causalidad circular compleja entre los niveles micro y macro en ciencias sociales es la ecuación master para la evolución de las distribuciones que representan los valores de las variables macrosociales. Las distribuciones $f(\mathbf{x})$ representarían las distintas inversiones de los (distintos) individuos en $\mathrm{N}$ actividades grupales, por ejemplo, calorías gastadas en el conjunto $\mathrm{x}$ de prácticas por unidad de tiempo, donde $\mathrm{x}=\left(x_{1}, x_{2}, \ldots, x_{\mathrm{N}}\right)$. Tales distribuciones representarían la no uniformidad individual dentro de una población, a la hora de invertir el esfuerzo en distintas actividades posibles.

La ecuación master se obtiene de la siguiente forma:

Representamos nuestro sistema en el espacio de estados como un vector $\mathrm{x}$. Sea $f(x, t)$ la probabilidad de encontrar a un individuo en el punto $\mathrm{x}$ en el tiempo $\mathrm{t}$, o lo que es equivalente, la fracción de individuos con respecto a la población total, que encontraríamos en ese punto en ese tiempo si hubiera posibilidad de repetir muchas veces el experimento. Vamos a ver cómo esta función densidad de probabilidad $f$ evoluciona en el tiempo.

La probabilidad $f(\mathrm{x}, t)$ se incrementará debido a transiciones desde otros puntos $x^{\prime}, y$ decrecerá debido a las transiciones que dejan el estado $x$, esto es, es la diferencia de dos tasas I e I':

$$
\frac{d P(\mathrm{x}, t)}{d t}=\mathrm{I}-I
$$

Ya que el término I incluye todas las transiciones del tipo $x^{\prime} \rightarrow x$, incluye la suma sobre todos los estados iniciales $\mathbf{x}^{\prime}$. Cada término de esa suma viene dado por la probabilidad de encontrar al sistema en $\mathbf{x}$ ' multiplicado por la probabilidad de transición por unidad de tiempo del paso $x^{\prime} \rightarrow x$, así:

$$
I=\Sigma_{\mathrm{x}} \cdot W\left(\mathrm{x}, \mathrm{x}^{\prime}\right) f\left(\mathrm{x}^{\prime}, t\right)
$$

donde $\mathrm{W}$ es la probabilidad de transición por unidad de tiempo.

Del mismo modo, para las transiciones «salientes» I' tenemos:

$$
I^{\prime}=f(\mathrm{x}, t) \Sigma_{\mathrm{x}}, \neq_{\mathrm{x}} W\left(\mathrm{x}^{\prime}, \mathrm{x}\right)
$$

De modo que la ecuación de balance, o ecuación master es:

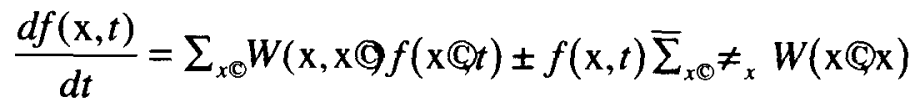


La ecuación «master», bajo la hipótesis de que todas las transiciones se producen atravesando estados vecinos, puede ser escrita en general en la forma de una ecuación de Fokker-Planck, que tiene la forma siguiente:

$$
\frac{\partial f(\mathrm{x}, t)}{\partial t}= \pm \sum_{i=1}^{N} \frac{\partial}{\partial x_{i}}\left[d_{i}(\mathrm{x}, t)\right]+\frac{1}{2} \sum_{i, j=1}^{N} \frac{\partial^{2}\left[S_{i j}(x, t) f(x, t)\right]}{\partial x_{i} \partial x_{j}}
$$

con $k_{i, k} k_{j} \in\{ \pm 1\}$, dado que sólo se permiten transiciones entre los estados vecinos más próximos.

La ecuación de Fokker-Planck tiene una solución bien conocida (ecuación de Chapman-Kolmogorov:

$$
f(\mathrm{z}, t+\Delta t)=\int_{x} d x f(x, t) \sqrt{\frac{\operatorname{det}[S(y, t)]}{2 \pi^{N} \Delta t} \exp \left[\frac{[\mathrm{z} \pm \mathrm{y} \pm \mathrm{d}(\mathrm{y}, t) \Delta t]^{T}[\mathrm{z} \pm \mathrm{y} \pm \mathrm{d}(\mathrm{y}, t) \Delta t]}{ \pm 2 \Delta t}\right]}
$$

Aquí, $f(x, t)$ representa la distribución de las inversiones de los sujetos en actividades (no institucionalizadas) o prácticas (sí institucionalizadas) colectivas o configuración en el tiempo t. La función $\operatorname{Sij}(\mathrm{x})$ representa la dispersión que sufren por unidad de tiempo en torno a $x$ las aportaciones que contribuyen con una inversión $\mathrm{x}$ a la actividad colectiva, debido a efectos aleatorios o no-sistemáticos. La función vectorial $d(\mathrm{x})$ representa la variación sistemática por unidad de tiempo o atracción sistemática que sufre en promedio el grupo de aportaciones situadas en $x$ hacia otros estados distintos $x$ '.

$\mathrm{d}=\left(d_{1}, d_{2}, \ldots d_{N}\right)$ y $\mathrm{S}=\left[S_{i j}\right]$, llamados coeficiente de derivas y matriz de fluctuaciones, respectivamente, son pues las tasas de variación temporal de los dos primeros momentos de las subdistribuciones infinitesimales que podemos suponer constituyen a la distribución completa $\mathrm{f}$ :

$$
\begin{gathered}
d_{i}=\lim _{\Delta t \rightarrow 0} \frac{<\Delta x_{i}}{\Delta t} \\
S_{i j}=\lim _{\Delta t \rightarrow 0} \frac{<\Delta x_{i} \Delta x_{j}>}{\Delta t}
\end{gathered}
$$

Estos dos parámetros se pueden relacionar co las probabilidades de transición hacia estados vecinos más próximos $w_{\mathrm{x} \rightarrow \mathrm{x}+\mathrm{k}}$ mediante las expresiones:

$$
\begin{aligned}
& d_{i}(\mathrm{x}, t)=\sum_{\mathrm{k}} k_{i} w_{x+\mathrm{k} \rightarrow \mathrm{x}}(t) \quad i, j=1,2, \ldots N \\
& S_{i} j(\mathrm{x}, t)=\sum_{\mathrm{k}} k_{i} k_{j} w_{x+\mathrm{k} \rightarrow \mathrm{x}}(t) \quad k_{i}, k_{j} \in\{ \pm 1\}
\end{aligned}
$$

Si en lugar de aportaciones a actividades se tratara de individuos de una población, el significado sería aún más inmediato. El efecto de $\mathrm{d}(\mathrm{x})$ es entonces provocar 
una deriva sistemática promedio del grupo local que está en $\mathrm{x}$ hacia otros estados $\mathrm{x}$ ' y simula el efecto medio de las variables macrosociales y os parámetros del entorno sobre el comportamiento individual medio. El efecto de $S(x)$ es dispersar al grupo aleatoriamente en torno a su estado $x$ de partida y simula la intrínseca variabilidad del comportamiento individual, modelable sólo en términos estadísticos.

La figura 1 muestra el efecto que tendría un comportamiento aleatorio de exploración de los individuos (una S constante) sobre una distribución de individuos localizada en un punto. La varianza de la distribución crece proporcionalmente con el tiempo.

La figura 2 ilustra en cambio el efecto de una deriva sin término fluctuante $(\mathrm{S}=0)$ sobre la misma distribución inicial. En este caso se ha generado un atractor desde el principio escribiendo la deriva como menos el gradiente (derivada, pendiente) de una función cóncava. Como puede observarse, la distribución tiende asintóticamente en el tiempo a acercarse al punto en que está el fondo de la función potencial, que sería la posición atractora. Como $S=0$, la distribución no explora sus alrededores en este caso, sólo se traslada como un todo.

La figura 3 muestra la evolución temporal de una distribución situada ya en el entorno de un atractor estacionario cuando ese atractor se bifurca por causas externas. La distribución se hace bimodal, por lo que un individuo componente tiene la misma probabilidad de acercarse a cualquiera de los dos nuevos puntos atractores.

La resolución de la ecuación de Fokker-Planck permite seguir en el tiempo una configuración inicial de comportamientos micro en la variable $\mathrm{x}$ a partir de cualquier distribución inicial de la forma que se quiera.

La microconfiguración representada por una distribución de aportaciones a una práctica en un sistema tecnológico tentativo, estable o institucionalizado, tiene asociada una producción-de-variable-macro, que es una función emergente de la coacción microscópica. Un ejemplo sería la simple suma de las producciones micro, o la media móvil de dicha suma en los últimos 365 días.

En la ecuación de Fokker-Planck, todas las retroacciones «medias» que se dan sobre las acciones micro se modelan mediante una función determinista que relaciona la deriva media local con: (i) las variables y parámetros definiendo el entorno, (ii) el microestado o distribución de todas las aportaciones individuales y (iii) todas las emergencias macro de dicho microestado. Mientras que la intrínseca variabilidad de los constituyentes y las influencias no deterministas entre los constituyentes se modelan estocásticamente, esto es, de forma estadística, a través de la matriz de fluctuaciones y sus eventuales dependencias funcionales de los parámetros que definen el entorno del sistema y del propio microestado de nuevo.

Esta naturaleza dual del planteamieto es metodológicamente muy potente, pues permite separar el análisis en dos programas de investigación complementarios. El primer programa de investigación debe estudiar la procedencia e intensidad de la variabilidad intrínseca al comportamiento micro y cómo cuantificarla estadísticamente. El segundo programa de investigación debe tratar de establecer cuáles son las retroacciones principales que relacionan a los movimientos medios de todas las acciones microsociales con la situación de todas las acciones microsociales, con sus emergencias macro y con parámetros externos al sistema social. Muchas de las variables macro son promedios agregados de comportamiento micro. 
Es de esperar que las derivas sean fuertemente sensibles a las variables sociales agregadas y a los parámetros externos, a veces en forma no lineal. Cuando se introducen expresiones no lineales para las derivas, el comportamiento potencial del sistema se hace enormemente rico, pues, como discutimos en un trabajo previo (García-Olivares 1999-a), son posibles entonces fenómenos como los siguientes: (i) inestabilización de atractores por variación de los parámetros externos del sistema y aparición de nuevos atractores; (ii) inestabilización del antiguo atractor por interacción con nuevas variables (variables agregadas generadas por autoorganización o aparecidas por innovación tecnológica o interacción con nuevos grupos) y generación de nuevos atractores y nuevas variables macro relevantes; (iii)

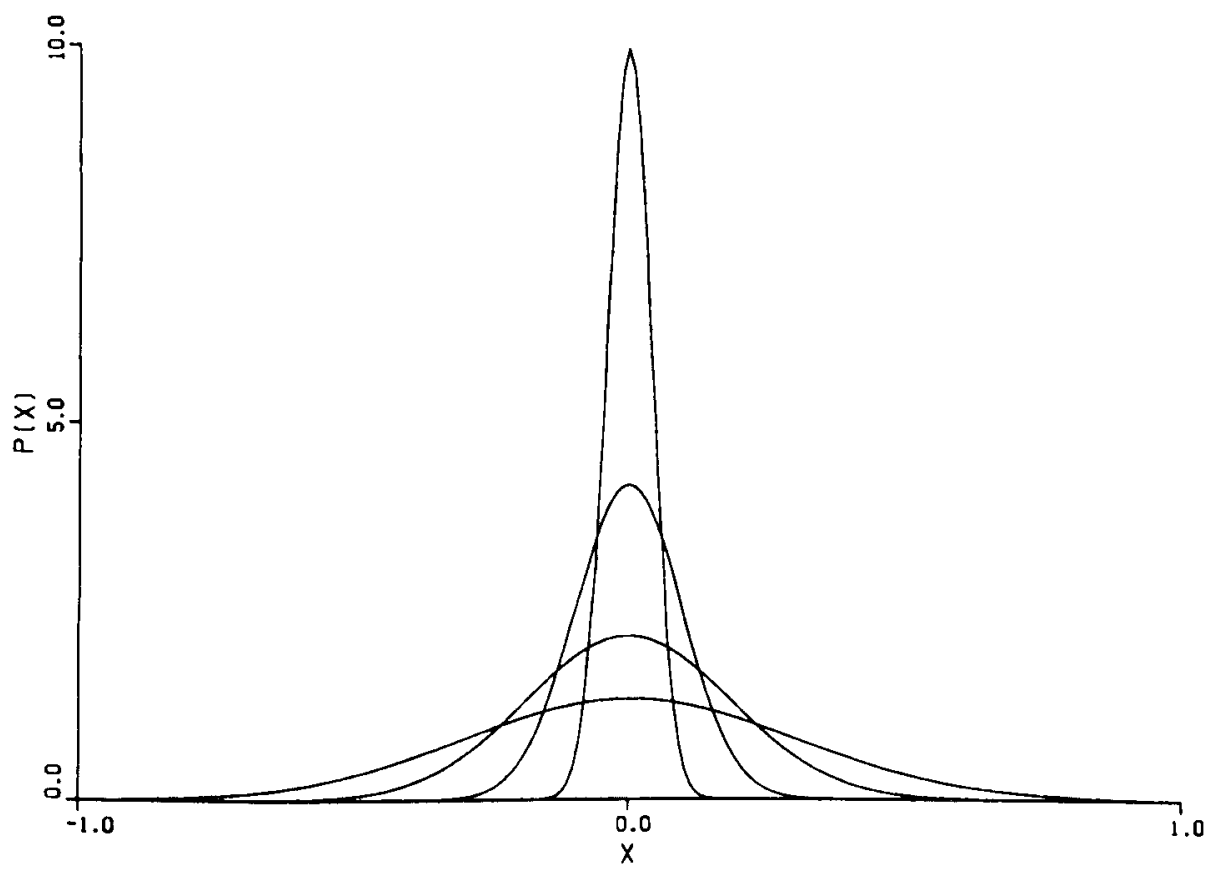

Figura 1. Efecto que tiene un comportamiento aleatorio de exploración (una $S$ constante) sobre una distribución de individuos localizada en un punto. $d=0, S=1$.

la posible generación de atractores caóticos; (iv) el ensamblaje de sistemas autoorganizativos en la forma de sistemas que se automantienen.

Además, la relación micro-macro es reestablecida en toda su generalidad, permitiendo que los comportamientos medios locales de los actores micro y las fluctuaciones locales de estos actores sean una función del microestado como un todo y sus emergencias, esto es, de funciones colectivas, no locales. De modo que cada una de las derivas debe ser a la vez una funcion del microestado y una función no lineal de las variables macro, sociales y no sociales. 


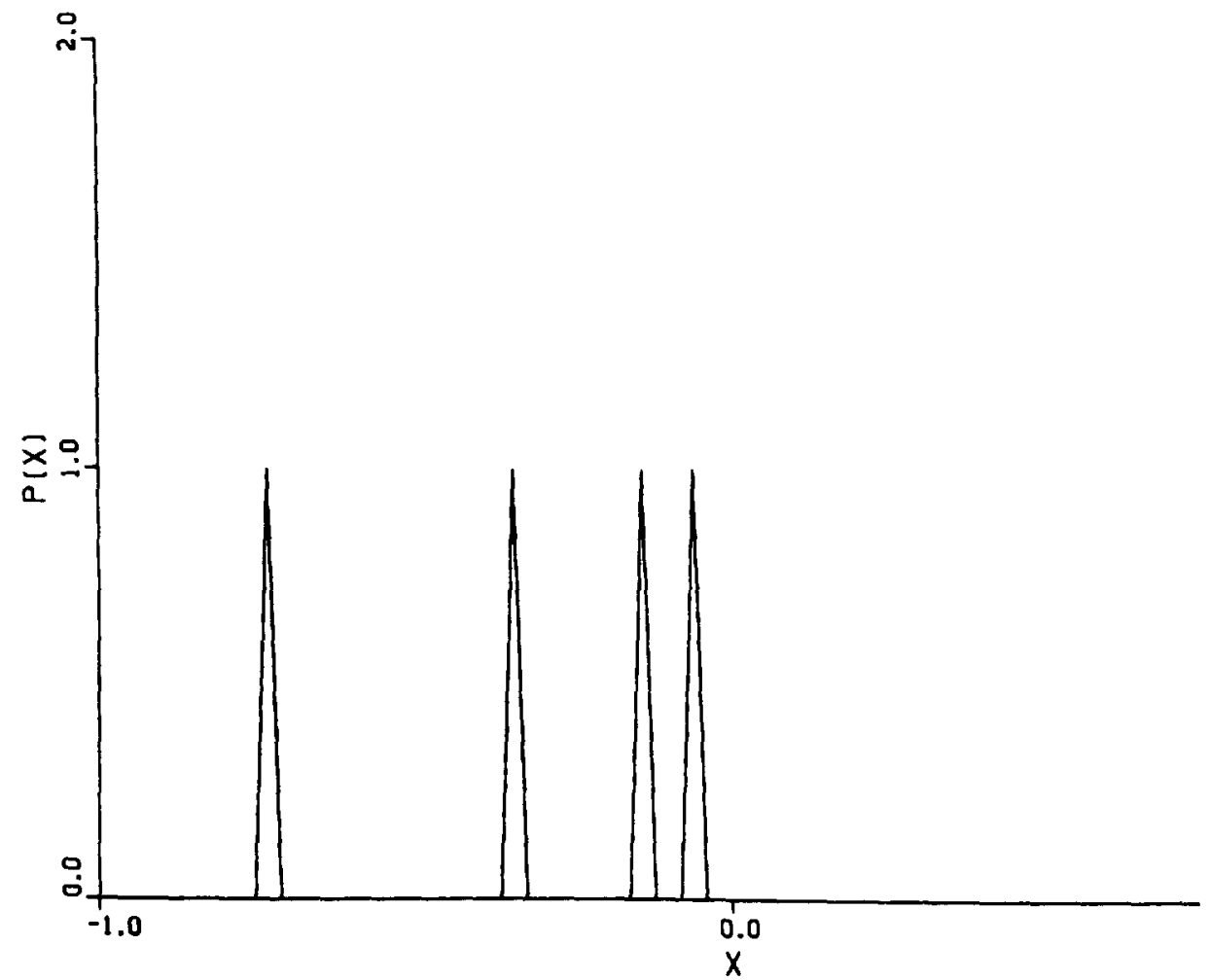

Figura 2. Efecto de una deriva sin término fluctuante ( $S=0)$ sobre una distribución localizada. Se ha generado un atractor desde el principio escribiendo la deriva como menos el gradiente de una función cóncava: $d= \pm \frac{d}{d x}\left(\frac{k}{2} x^{2}\right)= \pm k x$, con $\mathrm{k}=20$.

De este modo, se puede expresar también con toda naturalidad lo más esencial de un proceso autoorganizativo, que podemos definir del siguiente modo. Un proceso autoorganizativo es un proceso que tiene lugar (i) dentro de un sistema abierto constituído por muchas partes o agentes constituyentes que interaccionan; (ii) tales constituyentes intercambian flujos de energía, materiales y señales; (iii) tales intercambios son locales, pero a veces, por azar, y siempre que los intercambios tengan una intensidad suficiente, se sincronizan de tal modo que generan correlaciones entre subsistemas muy lejanos («organización», aumento de orden estructural); (iv) tales correlaciones producen propiedades macroscópicas observables («estructuras» nuevas o «emergencias» nuevas), (v) algunas de las cuales facilitan probabilísticamente el mantenimiento del conjunto de intercambios que ha sido capaz de producir la estructura recienaparecida («auto-organización», «automantenimiento», «autocatálisis», «autocircumscripción»).

Cuando la ecuación master incluye transiciones no sólo entre estados vecinos, sino también entre estados lejanos, entonces la ecuación que se obtiene no 


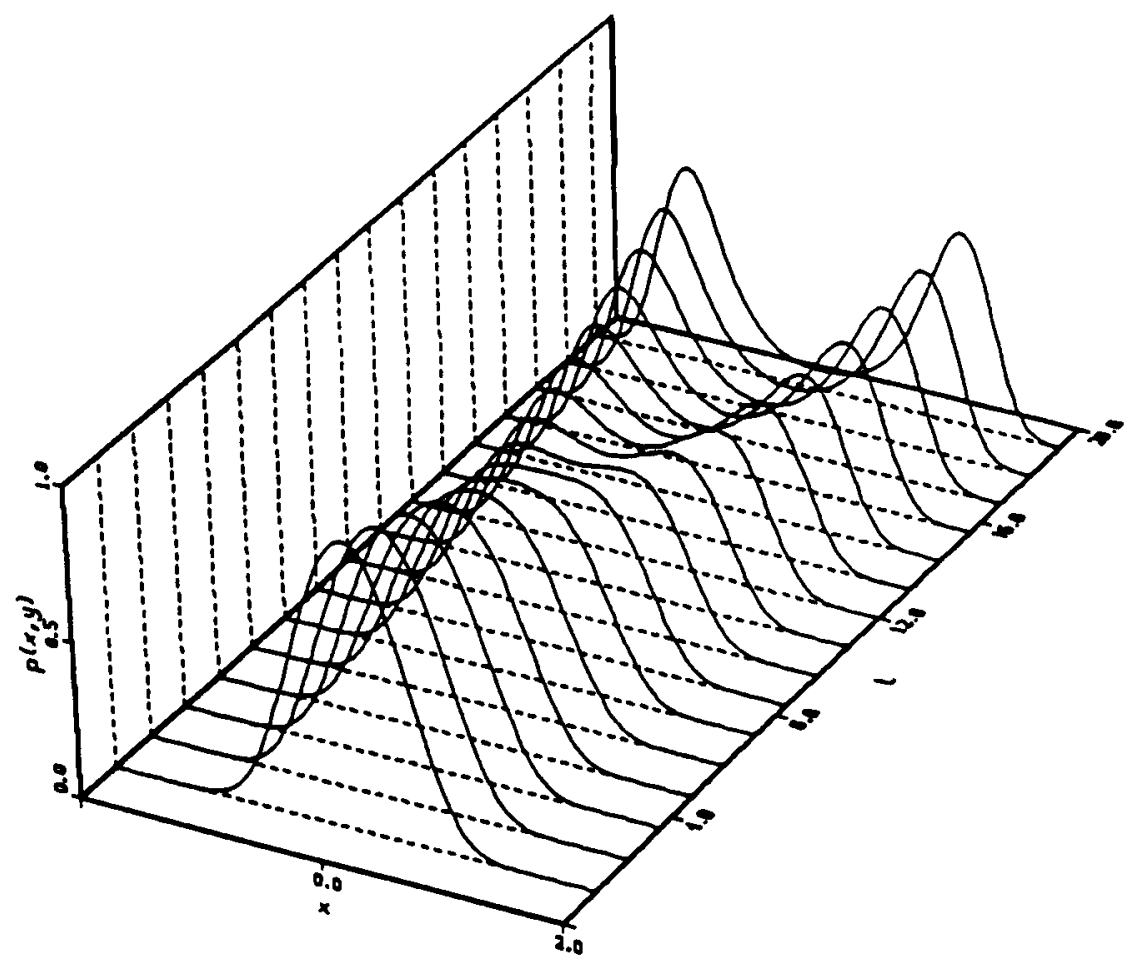

Figura 3. Evolución temporal de una distribución situada en el entorno de un atractor estacionario cuando ese atractor se bifurca por causas externas

$S=1$ y $d= \pm \frac{d}{d x}\left(\alpha x^{2} \pm \beta x^{4}\right)=10\left(\alpha x^{3}\right)$, con $\alpha=0.125 t-1$, donde $t$ es el tiempo. Cuando
$\alpha=0$ el atractor se bifurca.

es exactamente la de Fokker-Planck sino una algo más complicada. La ventaja de estas ecuaciones con transiciones lejanas es que pueden incorporar también perturbaciones y fluctuaciones estadísticas micro de varianza infinita, como las distribuciones de Lévy propuestas por Mandelbrot (Mandelbrot 1988) para la representación de algunos procesos de «azar salvaje».

\section{ESTUDIOS EN DINÁMICA DE GRUPOS, CIUDADES Y ECONOMÍA}

Modelos basados en las ecuaciones master y Fokker-Planck han sido ampliamente utilizados por Wedlich y Haag (Wedlich y Haag 1983) en la simulación de cambios de opinión en grupos de votantes, por Allen (Allen 1984) en la simulación del crecimiento de algunas ciudades, por Brian Arthur (Arthur 1988) en la simulación de «equilibrios puntuados» en economía y por Schweitzer y Schimansky-Geiger (Schweitzer y Schimansky-Geiger 1995) en la simulación de procesos colectivos dependientes del camino. 
Uno de los campos en que con mayor interés se estan empezando a aplicar los modelos que hemos estudiado es en el de la economía del no-equilibrio. Brian Arthur ha ilustrado un amplio conjunto de situaciones en economía en las que la existencia de equilibrios múltiples, dependencia del camino de las variables económicas y auto-refuerzo debido a feedbacks positivos en los beneficios contradicen las hipótesis habituales del equilibrio de mercado.

Las tecnologías de vídeo Sony Betamax y VHS generaron por ejemplo autoreforzamientos en sus respectivos mercados, en el sentido de que un incremento de la presencia en el mercado de una de las tecnologías estimula a los productores de cintas a producir más en ese formato; ello mejora los márgenes de beneficio de los adjudicatarios de esa tecnología, etc. Así, una pequeña ventaja producto de la suerte, de circunstancias externas o de las maniobras de los comerciales, puede generar un efecto catalítico irreversible de control sobre el $100 \%$ del mercado. Esto es lo que aparentemente consiguió VHS, pese a que muchos especialistas opinan que el perdedor, Betamax, es técnicamente superior a VHS. Como las tecnologías suelen mejorar con su adopción y su uso, el que una de ellas sea elegida antes que las otras puede ser decisivo a largo plazo, incluso aunque luego la decisión sea exclusivamente racional.

Un proceso de este tipo podría ser modelado con la ecuación master. La existencia en el mercado en dos técnicas con sus bondades específicas podría ser representada mediante una capacidad de atracción económica sobre los consumidores que en cada caso afectaría a las probabilidades de transición de los compradores hacia la adquisición de uno y otro sistemas. Así, la probabilidad de adquisición de cada uno de los sistemas sería proporcional a una constante (caracterizando a su bondad tecnológica particular y la curiosidad de los compradores) mas un término proporcional al número de consumidores que ya han comprado ese sistema.

Muchos análisis históricos, en teoría de las ideologías y en antropología usan implícita o explícitamente la hipótesis de que la elección y articulación de una cosmovisión, una religión, la dominación estatal, el dominio masculino, etc, han sido y son procesos de esta clase: contingentes al principio, y con ventajas que fueron in crescendo con su uso, y no una especie de ley de la naturaleza humana, ahistórica y determinable a priori. Por ello, el modelo del auto-refuerzo catalítico podría ser aplicado también a esos fenómenos de largo alcance.

Otra situación de auto-refuerzo deriva de las ventajas que una industria recibe de la presencia de otras industrias y servicios en sus cercanías. Ello induce a la formación de "parques» industriales y tecnológicos de forma espontánea, como una solución de coste global mínimo, en lugares que a veces no son los mejores, pero que son el producto de "accidentes históricos" precipitantes del proceso. Esta clase de dinámica ha sido aplicada por Allen al crecimiento probable de los barrios industriales, servicios y transportes de algunas ciudades como Bruselas (Allen 1984).

En la situación anterior tenemos un conjunto de actividades que se distribuyen aleatoriamente hasta que, por azar o circunstancias externas, en torno a una localización particular aparece una densidad de actividad lo suficientemente alta como para empezar a resultar atractiva como emplazamiento para nuevos actores que se añaden. 
La caída hacia el atractor continuará hasta un estado estacionario en que las caídas por atracción igualen las salidas debidas a la intrínseca variabilidad de los actores presentes (a lo cual se podría añadir una retroacción negativa determinista por efecto de las deseconomías del exceso de densidad).

Este proceso puede modelarse mediante una ecuación master en la que haya probabilidades de transición hacia otros estados proporcionales a la densidad de ocupación de tales estados receptores, y probabilidades de transición desde el propio estado hacia otro cualquiera igual a una constante (variabilidad individual) mas un término proporcional a la densidad del estado emisor. Esta segunda función tendría valores comparables a la primera únicamente para densidades muy altas. Hay que asegurarse de que la suma de las probabilidades de transición salientes de un estado hacia otros sea siempre menor que uno. En general, será mucho menor que uno, pues lo más probable para un agente particular es que, para intervalos temporales pequeños, permanezca en el estado en el que está.

Alternativamente, el proceso podría modelarse también mediante una ecuación de Fokker-Planck en la que la deriva local $d(\mathrm{x})$ sea la suma de dos términos: uno proporcional al gradiente de la concentración o densidad de actividad local y otro proporcional a menos el gradiente de la densidad de actividad local, siendo la constante de proporcionalidad del segundo término mucho menor que la del primero. La variabilidad individual estaría incorporada en la matriz de fluctuaciones de nuestra ecuación.

Ambas formulaciones matemáticas serían equivalentes y cualquiera que se elija constituiría un importante heurístico a la hora de acercar el esquema conceptual de lo que esta ocurriendo a datos de origen empírico.

La solución de tal proceso sería análoga a la que se muestra en la figura 4 , en la que un grupo inicialmente en una posición neutra explora su entorno por variabilidad individual, hasta encontrar dos localizaciones en que sus prácticas resultan catalizadas por circunstancias geográficas o por la presencia previa de grupos cuyas actividades son complementarias con las propias. En el estado estacionario final, la población queda circunscrita en torno a los centros de atracción o de autocatálisis únicamente.

La figura 6 muestra la solución de una variante más del problema anterior. En este caso, el entorno periférico de un emplazamiento se convierte de repente, por razones externas (políticas por ejemplo), en más atractivo que el centro para determinadas actividades industriales.

\section{SIMULACIÓN DE MOVILIZACIONES COLECTIVAS}

Para que las probabilidades de transición w hacia diferentes aportaciones a una actividad dada y hacia contribuciones en actividades alternativas dependan no sólo del estado $\mathrm{x}$ en que se esté y del valor de la distribución $\mathrm{f}$ en ese punto, sino que también pueda depender de la forma de la propia distribución $\mathrm{f}$ como un todo, es útil definir un conjunto de funciones o funcionales $\S$, en la forma siguiente:

$$
w=\S(f(x), x)=\S[f(f(x)), x]
$$


La funcional $f$ depende de la distribucion micro y representa las variables y funciones macro generadas por las actividades micro $f(x)$. Un ejemplo lo constituye el área bajo $\mathrm{f}(\mathrm{x})$ o cualquier función de esa área. Otro ejemplo nos lo proporcionarían la «función de poder» utilizadas por Charles Tilly (Tilly 1978) para relacionar los recursos invertidos (área de $\mathrm{f}(\mathrm{x})$ ) y los bienes colectivos obtenidos por un grupo en movilización.

Las funciones $\S$ pueden considerarse como las retroacciones de las funciones emergentes "producción macro" $f$ sobre los comportamientos individuales. Representan la influencia de los bienes colectivos generados por la práctica o actividad grupal sobre los comportamientos individuales. Tales bienes colectivos atraen, obligan o hacen relativamente menos costoso el acercamiento de los sujetos hacia cierto comportamiento coherente particular. Representa pues la atracción o capacidad de influencia subjetiva de las funciones y bienes colectivos producidos (en las condiciones macro dadas) sobre los comportamientos de los distintos individuos. El poder grupal y la atracción relativa de un sistema organizado de prácticas (sistema tecnológico) se complementan pues para hacer más o menos intenso el efecto de atracción-hacia un sistema tecnológico.

Las funcionales $f$ son como dijimos los bienes colectivos que surgen de la coacción (acción colectiva). Hay co-acciones estables e institucionalizadas como las prácticas económicas individuales (las ocupaciones: profesiones y oficios), igual que hay co-acciones desestabilizadoras como movilizaciones, revueltas y revoluciones.

En otro lugar (García-Olivares 1999-b) hemos analizado la clase de estudios psico-sociales que serían adecuados para la obtención de estas funcionales y funciones $f$ y $\S$, por lo que no lo repetiremos aquí.

Las situaciones históricas en que se produce lucha o competencia entre atractores o entre sistemas tecnológicos organizados pueden ser modelados mediante un cuadro como el que proponemos. Por ejemplo, las fluctuaciones y autoamplificaciones que conducen desde un conjunto de movilizaciones y consignas tentativas, a una reestructuración política revolucionaria. También las luchas entre sistemas o elementos tecnológicos alternativos que conducen a veces a la «extinción» de alguno de ellos. O bien las luchas políticas en general.

En estos casos, harán su aparición prácticas colectivas nuevas, inicialmente minoritarias, o «exo-prácticas» alternativas a las dominantes y ensayadas. La probabilidad de transición hacia tales «comportamientos tentativos» sería en general función de las emergencias generadas por la distribución y del tamaño de la aportación tentativa de esfuerzos que se ha atrevido a ensayar el grupo en las exo-práctica (Figura 6).

Supongamos que tenemos la competencia entre una práctica colectiva establecida y otra tentativa ligada a un nuevo sistema tecnológico, una movilización política, o una nueva asociación. En primer lugar habría que suponer que las probabilidades de transición entre aportaciones menores y mayores son una funcion de alguna producción colectiva emergente. Las siguientes hipótesis de trabajo son un ejemplo de un posible comienzo de modelización heurística: 

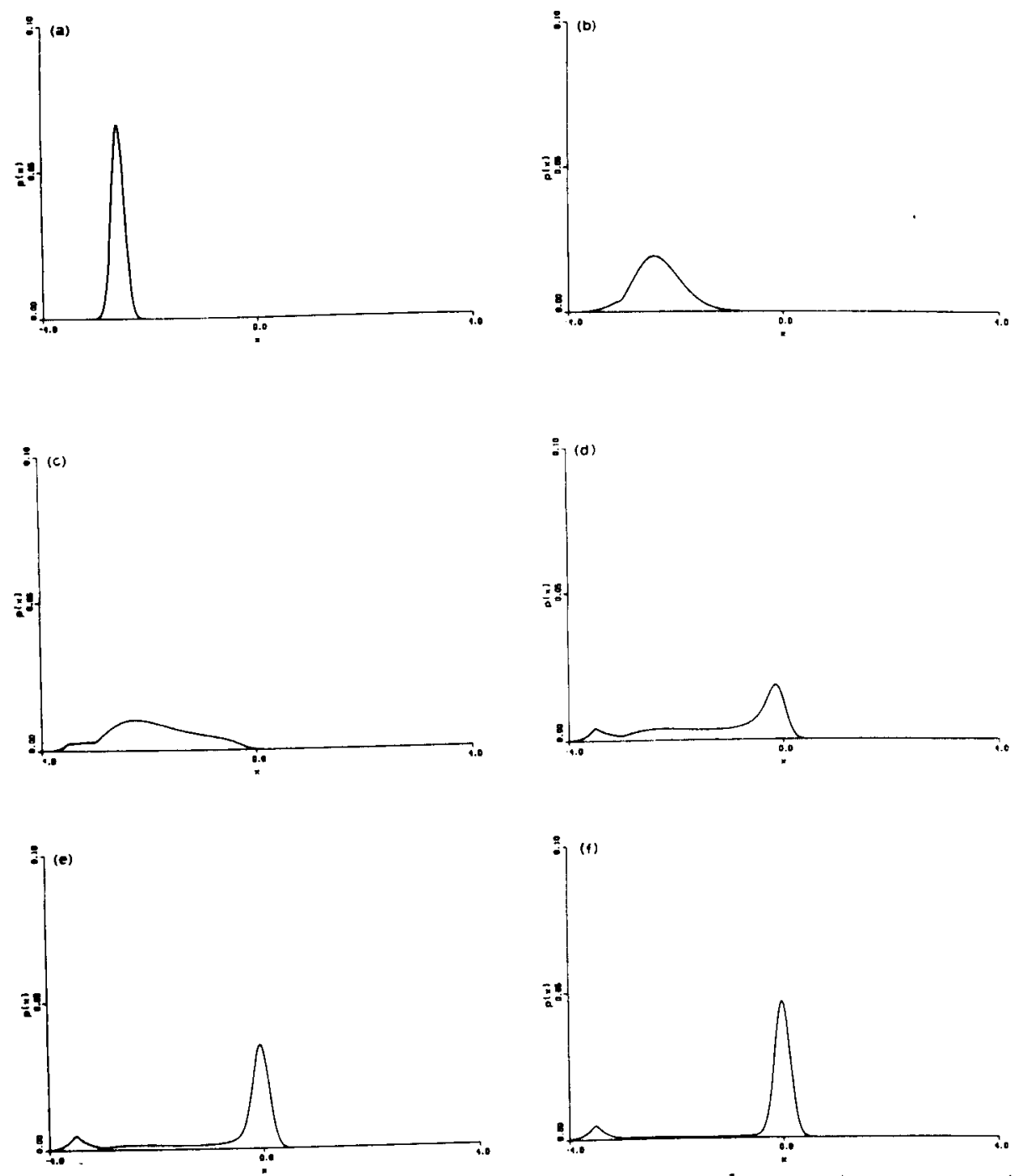

Figura 4. Un grupo, inicialmente en la posición neutra «a» explora su entorno y encuentra dos lugares que proporcionan una mayor atracción que la posición inicial.

A) La probabilidad de transición por unidad de tiempo $w\left(x_{1}, x_{2} \rightarrow x_{1}, x_{2}+1\right)$ desde una contribución individual $\left(x_{1}, x_{2}\right)$ hasta otra contribución que ha incrementado la aportación a la actividad 2 en una unidad, es tanto mayor cuanto menor sea la inversión previamente comprometida por el agente en la práctica 1 , esto es, menor sea $x_{1}$.

B) La probabilidad de que un agente aumente en una unidad su aportación a la práctica 2 es proporcional al esfuerzo total ya invertido por la población en la 


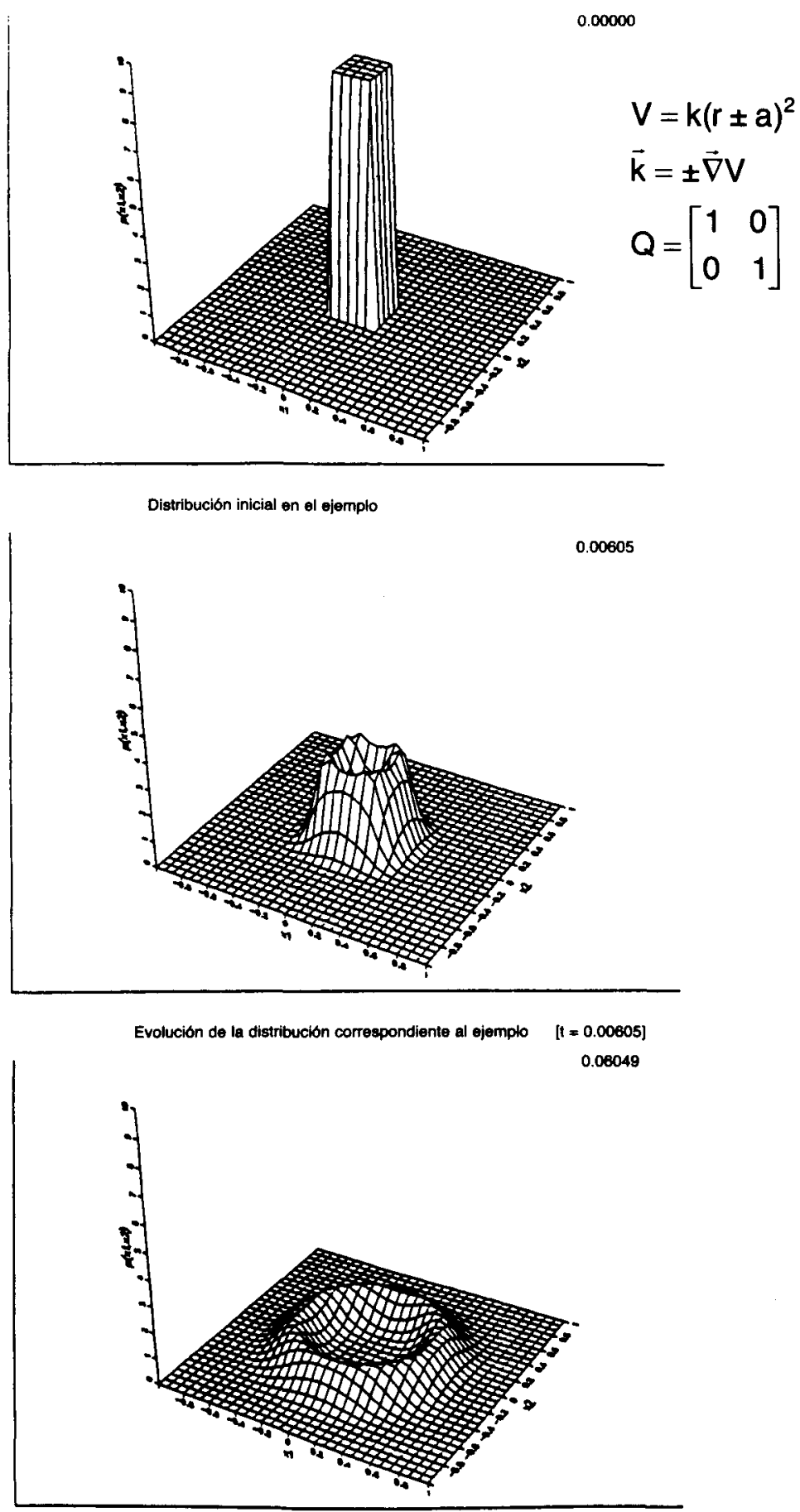

Figura 5. 
práctica $2, i_{2}$ (e inversamente proporcional a la inversión personal ya realizada en la práctica 1 , por $\mathrm{A})$ :

$i_{2}=E<x_{2}>=E \iint x_{2} f\left(x_{1}, x_{2}\right) d x_{1} d x_{2}$, siendo $\mathrm{E}$ el esfuerzo total (e.g. horas de trabajo) agregado invertido por la población $\mathrm{y}$ :

$w_{12}\left(x_{1}, x_{2} \rightarrow x_{1}, x_{2}+1\right) \alpha i_{2} / x_{1}$.

C) La probabilidad por unidad de tiempo de que un individuo aumente su aportación a la endopráctica (exopráctica) en una unidad es proporcional al cociente entre bienes colectivos obtenidos por la endopráctica (exopráctica) e inversión total realizada en ella por la población:

$w\left(x_{1}, x_{2} \rightarrow x_{1}, x_{2}\right) \alpha \S\left(i_{1}\right) i_{1}$

$w\left(x_{1}, x_{2} \rightarrow x_{1}, x_{2}+1\right) \propto \S\left(i_{2}\right) / i_{2}$

D) Las expresiones de los dos apartados anteriores serían ahora renormalizadas, de modo que sean compatibles unas con las otras, hasta obtener expresiones funcionales definitivas para las probabilidades de transición que sean verosímiles entre cada par de estados posibles.

E) Debe comprobarse el modelo obtenido para una ocupación inicial nula de la exopráctica: En ese caso debe obtenerse un estado estacionario realista para $f\left(x_{1}, 0\right)$. Debe comprobarse también que una ocupación azarosa inicial de la exopráctica con un número pequeño de aportaciones se basta para generar bienes colectivos que atraigan aportaciones adicionales hacia la nueva práctica. Si no fuera así, la forma funcional de las probabilidades de transición está mal definida y deberíamos volver de nuevo al paso A.

Otra interesante posibilidad en esta clase de modelos es definir la decisión que va a generar el agente en función de: (i) su posición local y (ii) la lista de reglas de decisión que mejor le han funcionado en el pasado según unas determinados criterios de juego. Tal lista de reglas va siendo modificada en el tiempo por un algoritmo genético que puntúa a las reglas componentes según su utilidad pasada y permite la sustitución de las peores por un conjunto de reglas nuevas generadas mediante mutación y recombinación de las mejores. Modelos de esta clase han sido utilizados por Palmer et al (1994), del Instituto de Santa Fe, para simular el comportamiento inteligente de los agentes y su adaptación permanente a la situación económica del mercado de capitales.

Esta clase de modelos son potencialmente de gran importancia y complementarios a los modelos anteriormente estudiados, debido a que la adaptaciónselección por el medio es el otro gran proceso evolutivo no tenido en cuenta directamente por los modelos basados en procesos autoorganizativos.

Además, el permitir la modificación de las reglas locales de interacción entre los agentes micro abre un mundo mucho más amplio a la creación de pautas nuevas, análoga en algún modo a la que muestran los sistemas con códigos genéticos y los sistemas sociales, que usan reglas culturales no predefinidas completamente para la interacción de sus agentes. En efecto, en muchas simulaciones efectuadas de sistemas complejos con reglas adaptativas, se observa la evolución frecuente del sistema en interacción hacia comportamientos que estan «en el límite del caos» (Langton, 1986). Cuán genérico es éste comportamiento y en qué condiciones se produce es algo que queda aún por delimitar. 


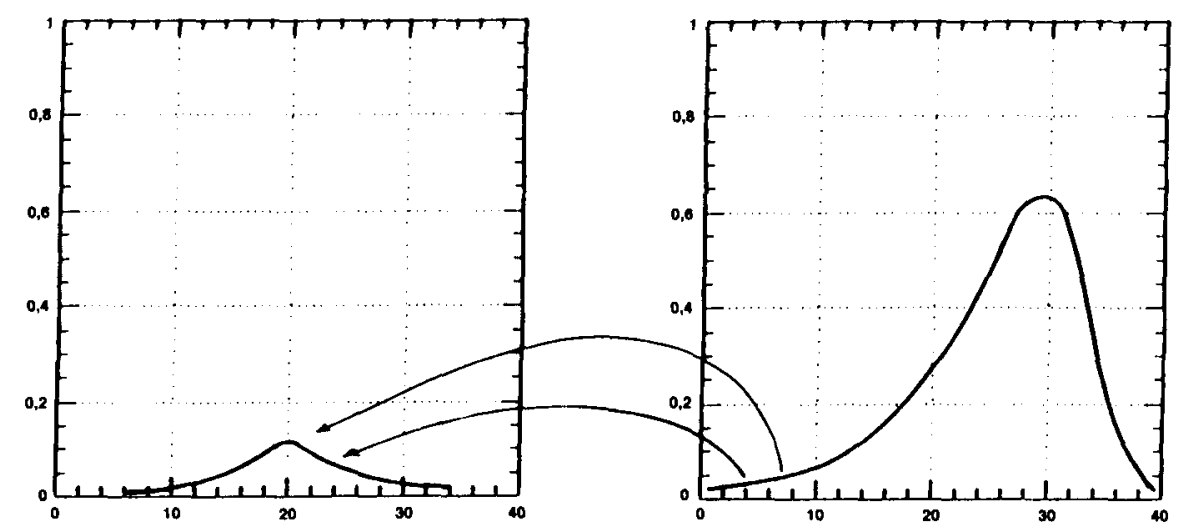

Figura 6. Competición entre dos prácticas colectivas alternativas. La figura muestra algunas transiciones desde la «endo-práctica» hasta una «exo-práctica» tentativa en desarrollo. Las curvas representan distribuciones de inversiones en cada una de las prácticas.

\section{CONCLUSIONES Y RETOS FUTUROS}

En este trabajo hemos estudiado algunos de los modelos matemáticos actualmente en uso en los programas de investigación de los sistemas autoorganizativos, la sinergética y los agentes adaptativos artificiales, y hemos analizado el modo como podrían ser aplicados a algunos problemas fundamentales de las ciencias sociales, en particular, la coevolución de los niveles microsocial y macrosocial.

Los nuevos paradigmas que se están introduciendo en la ciencia moderna desde los trabajos pioneros de Ilya Prigogine están contribuyendo de manera fundamental a la descripción cualitativa y cuantitativa de procesos de cambio en sistemas complejos mediante metáforas y procedimientos no reduccionistas y de cierto realismo. Ello está estimulando en gran manera el replanteamiento noreduccionista y no-simplista de muchos procesos dinámicos y de cambio social dentro del marco de las ciencias sociales. En primer lugar porque contempla desde un esquema inmanente y concreto los dos niveles de análisis y existencia, micro y macro. En segundo lugar, porque pone las bases de un lenguaje interdisciplinario que puede abarcar potencialmente desde las ciencias denominadas «duras» hasta disciplinas que hasta ahora se han expresado sólo en términos cualitativos.

Sin embargo, a la hora de aplicar modelos de esta clase a procesos de cambio social conviene ser consciente de una limitación intrínseca de la modelización. Los modelos utilizan conjuntos de metáforas culturalmente dominantes, sobre cómo está parcelado el mundo, cómo se relacionan los entes entre sí, qué procesos son cognoscibles, qué cambios pueden ser predichos y cuáles no, y qué clase de utilidad práctica tiene la modelización. Los mismos conceptos de mecanismo, causa determinista y variabilidad estocástica son también metáforas, muy útiles en el actual contexto científico y económico pero metáforas que eventualmente pueden ser modificadas en significado o sustituídas por otras nuevas, tras un cambio social que introduzca metáforas nuevas no del todo compatibles con las previas. 
Cuando un proceso autoorganizativo a nivel social genera sujetos colectivos capaces de instituir sistemas tecnológicos nuevos, instituciones nuevas y formas de dominación nuevas, emergentes estabilizados de esa sincronización colectiva previa, hay una última fase inevitable en el proceso que es la producción del significado del proceso.

A medida que van apareciendo emergencias inéditas del proceso, hace falta que alguien enuncie ( $y$ anuncie): «esto funciona», «esto es», y que esa identificación de las emergencias como relevantes sea aceptada por el uso social. Tal enunciado es metafórico, pues toda emergencia es impermanente y se basa en la persistencia de la acción colaboradora de los sujetos y de la permanente identificación de su nombre como relevante a sus usos. A la vez, tal enunciado metafórico fundante es también una reificación fetichista de lo que no es sino un conjunto de acciones micro persistentes.

Cuando una emergencia es resultado de prácticas sincronizadas nuevas o de sinergias complejas de prácticas muchas de ellas no institucionalizadas y no ensayadas previamente, la emergencia, inédita y sorprendente, puede no contar con términos adecuados para su descripción. Por ello, la metáfora identificadora tiende a articularse con las metáforas, mitos y discursos previamente disponibles. Por ejemplo, la desaparición de las poblaciones indígenas centroamericanas tras la llegada de los españoles fué interpretada por algunos intelectuales europeos como prueba de la virtud y aprobación divina de la implantación cristiana en el seno del paganismo y la barbarie. Hoy en día es interpretada en cambio como consecuencia de una combinación de desplazamiento cultural y de enfermedades epidémicas transmitidas por los europeos.

Sin embargo, al articularse la metáfora identificadora nueva con otras antiguas, tiende a alterarlas, al modificar en algún grado la extensión de sus significados, por lo que algunas de las metáforas previas podrían quedar seriamente debilitadas tras la aparición de la nueva. Hay en la historia cambios sociales, políticos y técnicos que generan auténticas revoluciones culturales, con la consiguiente modificación del sistema de metáforas dominante. Es de esperar que cambios de este calibre sigan produciéndose en un futuro que cada vez es más acelerado. Sin embargo, a la hora de predecir tales cambios, es conveniente ser consciente de que cambios de esa calibre podrían afectar a alguna de las metáforas que estamos utilizando ahora para simular el proceso.

La incorporación del cambio de los significados a los modelos que describen, mediante significados, el cambio social, es un último reto que esta clase de modelos de los procesos complejos deberían abordar tarde o temprano. Ello parece necesario si queremos que estos modelos esten al nivel de un objeto con una complejidad tan específica y abismal como la que tiene el objeto social.

\section{REFERENCIAS}

Allen, P. M., Sanglier, M. and Engelen, G. (1984): «Chance and Necessity in Urban Systems», in P. Schuster (de.): Stochastic Phenomena and Chaotic Behaviour in Complex Systems, Springer Verlag, p. 231-249. 
Arthur, W. Brian (1988): «Self-Reinforcing Mechanisms in Economics», en P. W. Anderson, K. Arrow y D. Pines (eds.): The economy as an evolving complex system, Reading, MA, Addison-Wesley 1988, pp. 9-31.

Garcta-Olivares, A. (1993): «Self-Organization and Intermittency in Social Systems: Towards a Science of Complexity», en Kybernetes 22, pp. 9-19.

- (1994): «Fokker-Planck equations in the simulation of complex systems», in Mathematics and computers in simulation 36, pp. 17-48.

- (1999-a): «La evolución de la complejidad», Enviado a Revista Empiria.

- (1999-b): «Un modelo matemático de la relación entre los niveles micro y macrosocial», a ser publicado en Nuevos avances en investigación social, Revista Anthropos.

HaKEN, H. (1987): Advanced Synergetics, Springer Verlag, Berlin. Véase también la amplia serie de títulos sobre Synergetics en Springer-Verlag.

Langton, Chris (1986): «Studying Life with Cellular Automata», Physica 22D, pp. 120 149.

Mandelarot (1988): Los Objetos Fractales, Ed. Tusquets.

G. Nicolis and I. Prigogine (1977): Self-Organization in Non-equilibrium Systems, Wiley. La ecuación master es estudiada en capítulos 9.3 y 10.

Palmer, R. G., W. Brian Arthur, John H. Holland, Blake LeBaron, Paul Tayler (1994): «Artificial economic life: a simple model of a stockmarket», Physica $D$ 75, pp. 264274.

SANTA Fé (1999): Véanse los Santa Fe Institute Studies in the Sciences of Complexity, Addison-Wesley, Reading, MA.

Schweitzer, Frank y Lutz Schimansky-Geier (1994): «Clustering of Active Walkers: Phase Transition from Local Interactions», Physica A 206, p. 359-379.

TilLy, Charles (1978): From Mobilization to Revolution, Addison-Wesley, Véase página 229 y ss.

W. Weiduchl\& G. HAAG, (1983): Concepts and Models of a Quantitative Sociology, Springer.

\section{RESUMEN}

En este trabajo tratamos de dar forma matemática operativa a algunos de los conceptos generados por la incipiente teoria de sistemas complejos con el fin de ilustrar el modo como pueden generarse modelos de utilidad heurística para la descripción y simulación de la relación entre procesos microsociales y macrosociales y su co-evolución mutua. Algunos de estos conceptos son: (i) la autoorganización por aparición de atractores en sistemas disipativos abiertos, (ii) la inestabilidad del sistema autoorganizativo ante cambios en los parámetros ambientales, $o$ ante la aparición de nuevas interacciones, (iii) la retroacción mutua entre los procesos microscópicos y sus emergencias macroscópicas y (iv) la coevolución adaptativa de las reglas de interacción utilizadas por sistemas agentes en concurrencia con otros sistemas agentes.

Se revisan algunas de las principales aplicaciones que se han hecho de estos conceptos en Ciencias Sociales, así como las herramientas matemáticas necesarias de cara a sus posibles aplicaciones prácticas en forma de modelos de simulación de procesos de cambio estructural, evolución tecnológica o social y agentes adaptativos. Finalmente, se proponen algunas metodologías de investigación útiles para construir modelos que tengan en cuenta la relación mutua entre los niveles micro y macrosocial. 


\begin{abstract}
Our objective in this paper is to translate in mathematically operative terms some of the concepts contributed by the recent complexity theory in order to illustrate the construction of usefull heuristic models of the coevolution of micro and macro social processes. Some of these processes are: (i) Selforganization by attractors generation in open dissipative systems, (ii) instability of the selforganizing system driven by either environmental parameters modification or apparison of new interactions, (iii) mutual feedback between microscopic processes and macroscopic emergencies and (iv) adaptive coevolution of interaction rules which are used by the agents in their interplay with another agents.

Some of the main reported applications of these concepts in social sciences are reviewed, as well as the mathematical methods needed to practical modelization purposes, with special emphasis in simulation of structural change, technological and social evolution and adaptive agents. Finally, some research methodologies are proposed which can be usefull to include micro-macro relationships in models of social evolution.
\end{abstract}

